\begin{tabular}{|c|c|}
\hline Title & $\begin{array}{l}\text { Enantioselective } 1,4 \text { addition of ary lboronic acids to } \alpha, \beta \text {-unsaturated carbonyl compounds catalyzed by rhodium( } I \text { - } \\
\text { chiral phosphoramidite complexes }\end{array}$ \\
\hline Author(s) & Kurihara, Kazunori; Sugishita, Noriyuki; Oshita, Kengo; Piao, Dongguo; Y amamoto, Y asunori; Miyaura, Norio \\
\hline Citation & $\begin{array}{l}\text { Journal of Organometallic Chemistry, 692(1-3), 428-435 } \\
\text { https://doi.org/10.1016/.jorganchem.2006.04.042 }\end{array}$ \\
\hline Issue Date & 2007-01-01 \\
\hline Doc URL & http:/hdl.handle.net/2115/18877 \\
\hline Type & article (author version) \\
\hline File Information & JOC692_1-3.pdf \\
\hline
\end{tabular}

Instructions for use 


\title{
Enantioselective 1,4-addition of arylboronic acids to $\alpha, \beta$-unsaturated carbonyl compounds catalyzed by rhodium(I)-chiral phosphoramidite complexes
}

\author{
Kazunori Kurihara, Noriyuki Sugishita, Kengo Oshita, Dongguo Piao, \\ Yasunori Yamamoto* and Norio Miyaura* \\ Division of Chemical Process Engineering, Graduate School of Engineering, \\ Hokkaido University, Sapporo 060-8628, Japan
}

\begin{abstract}
A chiral bidentate phosphoramidite (5a) was synthesized from Shibasaki's linked-(R)-BINOL and $\mathrm{P}\left(\mathrm{NMe}_{2}\right)_{3}$ as a new ligand for rhodium(I)-catalyzed asymmetric 1,4-addition of arylboronic acids to $\alpha, \beta$-unsaturated carbonyl compounds. The effects of $\mathbf{5 a}$ and Feringa's monodentate phosphoramidite $\left(4, \mathrm{R}^{1}, \mathrm{R}^{2}=\mathrm{Et}\right)$ on the yields and enantioselectivities were fully investigated. The reaction was significantly accelerated in the presence of a base such as $\mathrm{KOH}$ and $\mathrm{Et}_{3} \mathrm{~N}$, allowing the reaction to be completed at the lower temperatures than $50{ }^{\circ} \mathrm{C}$. The addition to cyclic enones such as 2-cyclopentenone, 2-cyclohexenone and 2-cycloheptenone at $50{ }^{\circ} \mathrm{C}$ in the presence of an $\left[\mathrm{Rh}(\mathrm{coe})_{2} \mathrm{Cl}\right]_{2}-4\left(\mathrm{R}^{1}, \mathrm{R}^{2}=\mathrm{Et}\right)$ complex resulted in enantioselectivities up to $98 \%$, though it was less effective for acyclic enones (0-70\% ee). On the other hand, a complex between $\left[\mathrm{Rh}(\mathrm{nbd})_{2}\right] \mathrm{BF}_{4}$ and 5a completed the addition to cyclic enones within $2 \mathrm{~h}$ at room temperature in the presence of $\mathrm{Et}_{3} \mathrm{~N}$ with 86-99\% yields and 96-99.8\%ee. This catalyst was also effective for acyclic enones, resulting in $62-98 \%$ yields and 66-94\%ee. The 1,4-additions of arylboronic acids to unsaturated lactones and acyclic esters with rhodium(I)-phosphoramidites complexes were also investigated.
\end{abstract}

Key words: arylboronic acids, rhodium catalyst, phosphoramidite, asymmetric, conjugate addition

\section{Introduction}

Metal-catalyzed conjugate addition reactions of carbon nucleophiles to $\alpha, \beta$-unsaturated compounds are the most widely used methods for asymmetric carbon-carbon bond formation [1]. The reactions catalyzed by copper [2], rhodium [3], and palladium [4] complexes are of great value for asymmetric syntheses because of the availability of chiral ligands. Rhodium(I)-binap catalysts were found to be excellent catalysts for 1,4-addition reactions of aryl- and 1-alkenylboronic acids to 
electron-deficient alkenes [3, 5]. Other catalysts that are effective for arylboronic acids are rhodium(I) complexes of mono-phosphoramidites [6], chiral P-P ligands such as chiraphos [7] and diphosphonites [8], P-N ligands of amidomonophosphines [9], bis(alkene) ligands based on a norbornadiene skeleton [10], and carbene ligands derived from biscyclophane imidazolium salts [11]. Among these chiral auxiliaries for metal-catalyzed conjugate additions, phosphoramidites developed by Feringa [6, 12], such as 4 , are the only ligands for which monodentate form exhibit high enantioselectivity for a large number of asymmetric transformations, including copper- and rhodium-catalyzed conjugate addition of organozinc and -boron compounds, though the efficiency of a bidentate ligand bridged by diamine [12a] was reported in copper-catalyzed reaction of diethylzinc with cyclic enones. Herein we report the performance of monodentate and bidentate phosphoramidite ligands for asymmetric addition of arylboronic acids to $\alpha, \beta$-unsaturated carbonyl compounds (Scheme 1) [6a, 13]. A bidentate bisphosphoramidite 5a newly synthesized from Shibasaki’s linked-BINOL was found to be an excellent ligand for both cyclic and acyclic enones.

$<<$ Scheme $1>>$

\section{Results and discussion}

\subsection{Monodentate phosphoramidites for asymmetric 1,4-addition to enones}

The effects of monodentate phosphoramidite ligands (4) on 1,4-addition of arylboronic acids to enones are summarized in Table 1 . The catalyst was prepared in situ by mixing $\left[\mathrm{Rh}(\mathrm{coe}){ }_{2} \mathrm{Cl}\right]_{2}(1.5$ mol\%) and four equivalents of $\mathbf{4}$ at room temperature for $1 \mathrm{~h}$. The addition of arylboronic acid, enone, and aqueous $\mathrm{KOH}$ was then followed at room temperature. After being stirred for $6 \mathrm{~h}$ at 50 ${ }^{\circ} \mathrm{C}$, the products were isolated and analyzed by a chiral stationary column. Since the enantioselectivity was reduced by raising the reaction temperature, the presence of a base was critical to carry out the reaction under mild conditions and to achieve high enantioselective. The reaction was completed within $6 \mathrm{~h}$ at $50{ }^{\circ} \mathrm{C}$ in the presence of 1 equivalent of $\mathrm{KOH}, \mathrm{K}_{3} \mathrm{PO}_{4}$ or $\mathrm{K}_{2} \mathrm{CO}_{3}$ in striking contrast to the reaction occurring at $90{ }^{\circ} \mathrm{C}$ in the absence of a base. $\mathrm{Rh}$ (acac)(coe) $)_{2}, \quad\left[\mathrm{RhCl}\left(\mathrm{C}_{2} \mathrm{H}_{4}\right)_{2}\right]_{2}, \quad[\mathrm{Rh}(\mathrm{OH})(\mathrm{cod})]_{2}$ and $\mathrm{Rh}(\mathrm{acac})\left(\mathrm{C}_{2} \mathrm{H}_{4}\right)_{2}$ also gave yields and enantioselectivities analogous to those of $\left[\mathrm{Rh}(\mathrm{coe})_{2} \mathrm{Cl}\right]_{2}$. The enantioselectivities dramatically changed in a series of $N, N$-dialkylamino derivatives for 2-cyclohexenone (entries 1-8). Among the ligands employed, $N, N$-diethylamine and morpholine derivative exhibited the best enantioselectivity (91\% ee, entries 2 and 8), and the selectivities were reduced by increasing the bulkiness of amino groups (entries $5-7)$. The diethylamino ligand $\left(4, \mathrm{R}^{1}, \mathrm{R}^{2}=\mathrm{Et}\right)$ was also effective for other cyclic 
enones such as 2-cyclopentenone (75-95\%ee, entries 9-11) and 2-cycloheptenone (94\%ee, entry 12), giving yields and selectivities comparable to those of 2-cyclohexenone. It is interesting that the substituents on aromatic rings significantly affected the enantioselectivity. 3-Methoxy-, 3-chloro, and 4-tolylboronic acid resulted in apparently higher enantioselectivities than that of phenylboronic acid (entries 2-4 and 9-11). The phosphoramidites derived from $(R)-(+)$-BINOL afforded (R)-3-phenylcyclohexanone for a series of dialkylamino derivatives (entries 1, 2, 5, 7, 8), though the diisopropyl derivative exceptionally gave an $S$ isomer with a very low selectivity (entry 6).

\section{$<<$ Table 1 >>}

In contrast to the excellent performance of the $N, N$-diethylamino ligand for cyclic enones, it was not effective for acyclic enones (entries 13-20). Acyclic enones such as (E)-3-nonen-2-one unfortunately resulted in a racemic product (entry 13). Since the reactions were very slow when the bulkiness of two alkyl groups of 4 were increased (e.g., $\mathrm{R}^{1}, \mathrm{R}^{2}=$ benzyl, i-propyl). A series of methylalkylamine derivatives was synthesized to optimize the best ligand (entries 14-20). The $N, N$-t-butylmethylamino derivative was found to result in 70\%ee (entry 20); however, none of the enantioselectivities were a practical level. The absolute configuration of product (3h) determined by specific rotations was reversed from $S$ to $R$ by increasing bulkiness of the ligand.

\subsection{Preparation of bidentate phosphoramidites and their rhodium(I) complexes}

The use of a rigid bidentate ligand can be critical to achieve high enantioselectivity for flexible acyclic substrates. Thus, bidentate bisphosphoramidites $\mathbf{5}$ were newly synthesized on the basis of linked-BINOL (6), which was obtained from optically active BINOL by the procedures of Shibasaki [14] (Scheme 2).

\section{$<<$ Scheme 2>>}

The two methods pioneered by Feringa [15, 12c] were used for conversion of linked-BINOL to the corresponding phosphoramidites (5). A mixture of $\mathrm{P}\left(\mathrm{NMe}_{2}\right)_{3}$ or $\mathrm{P}\left(\mathrm{NEt}_{2}\right)_{3}$ and an $(R, R)$-O-linked-BINOL (6) was refluxed in toluene in the presence of a catalytic amount of $\mathrm{NH}_{4} \mathrm{Cl}$ to give air and moisture-stable bisphosphoramidite 5a (74\%) and $\mathbf{5 b}$ (56\%). The protocol failed to give an $N, N$-diisopropyl derivative (5c). Thus, 5c was synthesized in $11 \%$ yield by a two-step method that involves chlorophosphonylation of 6 at $-60{ }^{\circ} \mathrm{C}$ and amidation with lithium diisopropylamide [12c].

The reaction of $5 \mathbf{a}$ with $\left[\mathrm{Rh}(\mathrm{nbd})_{2}\right] \mathrm{BF}_{4}$ in $\mathrm{CD}_{2} \mathrm{Cl}_{2}$ gave the desired $[\mathrm{Rh}(\mathbf{4 a})(\mathrm{nbd})] \mathrm{BF}_{4}(\mathbf{7 a})$ as a fine powder. ${ }^{31} \mathrm{P}$ NMR exhibited a single signal at $142.4 \mathrm{ppm}\left(\mathrm{d}, J_{\mathrm{Rh}-\mathrm{P}}=248.9 \mathrm{~Hz}\right.$ ), thus suggesting the intramolecular complexation of two phosphorous atoms to a rhodium metal center. 
The formation of a $1: 1$ complex was also confirmed by mass spectroscopy (FAB), which showed a molecular weight of $955.1913\left(\mathrm{M}^{+}-\mathrm{BF}_{4}\right)$. The corresponding neutral complex $[\mathrm{Rh}(\mathrm{Cl})(\mathbf{5 a})](\mathbf{7 b})$ was synthesized by analogous reaction of 5 a with $\left[\mathrm{Rh}(\mathrm{Cl})(\mathrm{coe})_{2}\right]_{2}$, which exhibited a single signal at 153.7 ppm (d, $\left.J_{\mathrm{Rh}-\mathrm{P}}=296.3 \mathrm{~Hz}\right)$. A mixture of $\left[\mathrm{Rh}(\mathrm{nbd})_{2}\right] \mathrm{BF}_{4}$ and $\mathbf{4 b}$ also gave a single signal (142.3 ppm, d, $J=248.9 \mathrm{~Hz}$ ) analogous to that of 5a, but 4c exhibited multiple signals at 24.8, 111.0 and $134.1 \mathrm{ppm}$ due to formation of a mixture of intra- and intermolecular coordination.

\subsection{Bidentate phosphoramidites for asymmetric 1,4-addition to enones}

The effects of 5, rhodium catalysts and bases in the reaction of 2-cyclohexenone and phenylboronic acid in aqueous 1,4-dioxane are shown in Table 2. The catalysts were prepared in situ by mixing a rhodium precursor and 10\% excess of 5 since they resulted in yields and enantioselectivities that were same as those of isolated complexes (7a, 7b). The neutral complex thus prepared from $[\mathrm{RhCl}(\mathrm{coe})]_{2}$ and 5 a did not catalyze the reaction (entry 1), but the reaction was initiated by addition of a base at $50{ }^{\circ} \mathrm{C}$ with yields increasing in the order of basic strength (entries 2-4). Finally, aqueous $\mathrm{KOH}$ was recognized to be the best base for a neutral catalyst (entry 4), as was previously demonstrated in analogous conjugated addition catalyzed by Rh(I)-phosphine complexes. A combination of a cationic rhodium(I) complex and 5 provided a much more active catalyst than a neutral one. The reaction was completed within $0.5 \mathrm{~h}$ at room temperature when $\left[\mathrm{Rh}(\mathrm{nbd})_{2}\right] \mathrm{BF}_{4}$ and $5 \mathbf{a}$ were used in the presence of $\mathrm{Et}_{3} \mathrm{~N}$ [5k] (entries 5 and 6). A perfect enantioselectivity that close to $100 \%$ ee was obtained at room temperature (entry 6). On the other hand, $N, N$-diethyl (5b) and $N, N$-diisopropyl (5c) derivatives were less effective than the $N, N$-dimethyl ligand (5a) (entries 7 and 8).

<Table 2>

The effects of catalyst amounts and reaction rates are shown in Figure 1. The addition of phenylboronic acid (1.5 equivalents) to 2-cyclohexenone completed within $30 \mathrm{~min}$ when a $3 \mathrm{~mol} \%$ of $\left[\mathrm{Rh}(\mathrm{nbd})_{2}\right] \mathrm{BF}_{4}-5 \mathrm{a}$ was used at $25{ }^{\circ} \mathrm{C}$. The use of 0.5 mol\% complex completed the reaction within $3 \mathrm{~h}$ at $50{ }^{\circ} \mathrm{C}$, though it was very slow at $25^{\circ} \mathrm{C}$. However, the reaction resulted in less than $5 \%$ yields even at temperatures higher than $50{ }^{\circ} \mathrm{C}$ when catalyst loading was reduced to $0.1 \mathrm{~mol} \%$.

$<$ Figure $1>$

With these optimized conditions, the scope of reaction was investigated by using representative arylboronic acids, $\alpha, \beta$-unsaturated carbonyl compounds, and an $\left[\mathrm{Rh}(\mathrm{nbd})_{2}\right] \mathrm{BF}_{4} / \mathbf{5 a}$ catalyst (Table 3). There was no difficulty in obtaining high chemical yields and high enantioselectivities for cyclic enones within $2 \mathrm{~h}$ at room temperature (entries 1-7). The catalyst was 
especially effective for 2-cyclohexenone, easily achieving over 99\%ee for various arylboronic acids (entries 1-4). The catalyst was less effective for acyclic enones; however, the selectivities were comparable to or even higher than those of mono-phosphoramidites $\mathbf{4}$ or previously reported bisphosphine ligands such as BINAP [5-11]. For example, the use of a catalyst obtained from 1/2[RhCl(coe) $]_{2}$ and 4 resulted in 0-70\%ee (Table 1, entries 13-20), and the use of Rh(I)-BINAP catalyst resulted in $83 \%$ ee for $(E)-\mathrm{C}_{5} \mathrm{H}_{11} \mathrm{CH}=\mathrm{CHCOCH}_{3}[5 \mathrm{k}]$, whereas $\left[\mathrm{Rh}(\mathrm{nbd})_{2}\right] \mathrm{BF}_{4} / 5$ a gave $74 \%$ ee at $25{ }^{\circ} \mathrm{C}$ (entry 8) and 84\%ee at $5{ }^{\circ} \mathrm{C}$ (entry 9). The enantioselectivities for acyclic $(E)$-enones were dependent on the $\beta$-substituent $\left(\mathrm{R}^{1}\right)$ and a substituent on ketone carbonyls $\left(\mathrm{R}^{3}\right)$. The effect of $\mathrm{R}^{1}$ increased in the order of $\mathrm{Ph}<n-\mathrm{C}_{5} \mathrm{H}_{11}<$ isopropyl for a series of methyl ketones (entries 10, 15 and 19). Steric balance between $\mathrm{R}^{1}$ and $\mathrm{R}^{3}$ was also an important factor affecting the selectivity. The selectivities were improved by increasing the bulkiness of $\mathrm{R}^{3}$ for enones having a primary alkyl group at the $\beta$-carbon (entries 10 and 13), but enones possessing a hindered substituent ( $\mathrm{R}^{1}=$ isopropyl and phenyl) reduced the selectivity by increasing the bulkiness of $\mathrm{R}^{3}$ groups $\left(\mathrm{CH}_{3}>\mathrm{Ph}>\right.$ cyclohexyl) (entries 15, 17, 18, 19 and 20). The para- and meta-substituents in arylboronic acids affected enantioselectivities (entries 8-12). The enantioselectivities of meta-substituted arylboronic acids were generally higher than that of para-substituted boronic acids, as shown in Table 1 and as previously reported for related rhodium- and palladium-catalyzed reactions $[4,5]$.

<Table 3>

\subsection{Asymmetric addition to $\alpha, \beta$-unsaturated esters}

Asymmetric 1,4-additions to acyclic and cyclic $\alpha, \beta$-unsaturated esters are shown in Scheme 3. The reaction was slower than that for enones, but high enantioselectivities comparable to those of the corresponding enones were easily obtained. Methyl crotonate, 5H-furan-2-one, and 5,6-dihydro-2H-pyran-2-one afforded 3v (75\%еe), 3w (77\%еe), 3x (89\%еe) and 3y (91\%еe), respectively, in the presence of $\left[\mathrm{Rh}(\mathrm{nbd})_{2}\right] \mathrm{BF}_{3}-5 \mathrm{a}$ and $\mathrm{Et}_{3} \mathrm{~N}$ at room temperature. On the other hand, the use of monodentate phosphoramidites such as $4\left(\mathrm{R}^{1}, \mathrm{R}^{2}=\mathrm{Et}\right)$ resulted in no reaction for $5 \mathrm{H}$-furan-2-one and 35\% yield and 72\%ee for 5,6-dihydro-2H-pyran-2-one at $50{ }^{\circ} \mathrm{C}$ in the presence of $\mathrm{Rh}(\text { aсас)(coe) })_{2}$ and $\mathrm{Et}_{3} \mathrm{~N}$ in dioxane- $\mathrm{H}_{2} \mathrm{O}(6 / 1)$.

$<<$ Scheme 3>>

\section{Conclusion}

In conclusion, the effects of catalysts, phosphoramidites $(4,5)$ and bases on reaction rates 
and enantioselectivities in the rhodium-catalyzed 1,4-addition of arylboronic acids to enones were investigated in detail. Although traditional monodentate phosphoramidite ligands (4) gave good enantioselectivities for cyclic enones, we have shown that bidentate phosphoramidite (5a), first prepared from Shibasaki's linked-BINOL, is an excellent ligand for both cyclic and acyclic enones and enable the reaction to be completed in a short time at room temperature. Works aimed at characterization of the catalysts by X-ray analysis are in progress to elucidate the enantioselection mechanism.

\section{Experimental}

\subsection{Reagents}

$\left[\mathrm{RhCl}(\text { coe })_{2}\right]_{2}[16]$ and $\left[\mathrm{Rh}(\mathrm{nbd})_{2}\right] \mathrm{BF}_{4}[17]$ were prepared by the reported procedures. Chiral phosphoramidites (4) were obtained from $(R)$-BINOL and the corresponding amines by the method of Feringa [15]. ( $R, R$ )-O-linked-BINOL (6) was synthesized from $(R)$-BINOL by the method of Shibasaki [14].

\subsection{Bidentate phosphoramidites (5, Scheme 2)}

\subsection{1. $N, N$-Dimethyl $(R, R)$-O-linked-phosphoramidite (5a)}

3,3"-(Oxydimethylene)-di-1,1'-bi-2-naphthol (6, $(R, R)$-O-linked-BINOL) (1 mmol), $\mathrm{NH}_{4} \mathrm{Cl}$ $(0.01 \mathrm{~g})$ and $\mathrm{P}\left(\mathrm{NMe}_{2}\right)_{3}(2.8 \mathrm{mmol})$ in dry toluene $(10 \mathrm{ml})$ were refluxed for $12 \mathrm{~h}$ under nitrogen. The crude solid obtained by evaporation of the solvent was crystallized from $\mathrm{CH}_{2} \mathrm{Cl}_{2}$ /pentane to give 5a as white crystals (74\%). ${ }^{1} \mathrm{H}-\mathrm{NMR}\left(400 \mathrm{MHz}, \mathrm{CD}_{2} \mathrm{Cl}_{2}\right)$ : $\delta=2.23-2.39(\mathrm{~m}, 12 \mathrm{H}), 4.82(\mathrm{~d}$, $J=13.3 \mathrm{~Hz}, 2 \mathrm{H}$ ), 5.02 (d, $J=13.3 \mathrm{~Hz}, 2 \mathrm{H}$ ), 7.07-7.39 (m, 14H), 7.76-7.86 (m, 6H), 8.15 (s, $2 \mathrm{H}) ;{ }^{13} \mathrm{C}$ NMR (100 MHz, $\left.\mathrm{CD}_{2} \mathrm{Cl}_{2}\right) \delta=35.7,35.9,69.2,122.1,122.9,124.2,125.1,126.2,126.5,126.8$, 127.0, 128.2, 128.7, 129.3, 130.6, 130.9, 131.0, 131.8, 132.3, 133.1, 148.0, 148.1, 149.7; ${ }^{31} \mathrm{P}$ NMR (161.7 Hz, $\left.\mathrm{CD}_{2} \mathrm{Cl}_{2}\right) \delta=$ 149.4; MS (m/z) 46 (33), 136 (31), 154 (41), 266 (27), 282 (47), 329 (100), 388 (25), 716 (28), $761\left(16,[\mathrm{M}+\mathrm{H}]^{+}\right)$; exact mass calcd for $\mathrm{C}_{46} \mathrm{H}_{38} \mathrm{~N}_{2} \mathrm{O}_{5} \mathrm{P}_{2}$ : 760.2256; found $760.2275 ;[\alpha]^{21}{ }_{\mathrm{D}}=-522.5^{\circ}\left(\mathrm{C}=0.56, \mathrm{CHCl}_{3}\right)$.

\subsection{2. $N$, $N$-Diethyl $(R, R)$-O-linked-phosphoramidite (5b)}

An analogous method used for preparation of $\mathbf{5 a}$ gave $\mathbf{5 b}$ in $56 \%$ yield. ${ }^{1} \mathrm{H}-\mathrm{NMR}$ (400 $\left.\mathrm{MHz}, \mathrm{CD}_{2} \mathrm{Cl}_{2}\right) \delta=0.75-0.91$ (m, 12 H), 2.64-2.95 (m, 8H), 4.89 (d, J=13.6 Hz, 2H), 5.06 (d, $J=13.6$ 
$\mathrm{Hz}, 2 \mathrm{H}), 7.09-7.41$ (m, 14H), 7.79-7.87 (m, 6H), 8.15 (d, J=8.8 Hz, 2H); ${ }^{13} \mathrm{C}$ NMR (100 MHz, $\left.\mathrm{CD}_{2} \mathrm{Cl}_{2}\right) \delta=15.0$, 39.1, 39.3, 69.1, 122.3, 124.3, 125.0, 125.1, 126.0, 126.4, 126.9, 127.1, 127.8, 128.7, 130.3, 130.6, 130.9, 131.2, 131.7, 132.3, 133.1, 148.4, 148.5, 149.9; ${ }^{31} \mathrm{P}$ NMR (161.7 MHz, $\left.\mathrm{CD}_{2} \mathrm{Cl}_{2}\right) \delta=150.1$; MS (m/z) 72 (31), 266 (38), 282 (51), 329 (100), 416 (15), 744 (28), 817 (10, $\left.[\mathrm{M}+\mathrm{H}]^{+}\right)$; exact mass calcd for $\mathrm{C}_{50} \mathrm{H}_{47} \mathrm{~N}_{2} \mathrm{O}_{5} \mathrm{P}_{2}\left([\mathrm{M}+\mathrm{H}]^{+}\right)$: 817.2961; found 817.2981; $[\alpha]^{22}{ }_{D}=-414.4$ $\circ\left(\mathrm{C}=0.50, \mathrm{CHCl}_{3}\right)$.

\subsection{3. $N, N$-Diisopropyl ( $R, R)$-O-linked-phosphoramidite (5c)}

To a mixture of $\mathrm{PCl}_{3}(2 \mathrm{mmol})$ and $\mathrm{Et}_{3} \mathrm{~N}(4 \mathrm{mmol})$ in toluene $(3 \mathrm{ml})$ was added a solution of 3,3"-(oxydimethylene)-di-1,1'-bi-2-naphthol (6, $(R, R)$-O-linked-BINOL) (1 mmol) in toluene $(10 \mathrm{ml})$ at $-60{ }^{\circ} \mathrm{C}$. After being stirred for $2 \mathrm{~h}$, the reaction mixture was allowed to warm up to room temperature. The precipitates were removed by filtration. The filtrate was treated with $n$-BuLi (2 $\mathrm{mmol})$ and $i-\mathrm{Pr}_{2} \mathrm{NH}(3 \mathrm{mmol})$ at $-40^{\circ} \mathrm{C}$. After being stirred for $16 \mathrm{~h}$ at room temperature, the crude solids obtained by evaporation of the solvent was crystallized from $\mathrm{CH}_{2} \mathrm{Cl}_{2}$ /pentane to give 5c as white crystals (11\%). ${ }^{1} \mathrm{H}-\mathrm{NMR}\left(400 \mathrm{MHz}, \mathrm{CD}_{2} \mathrm{Cl}_{2}\right) ; \delta$ 0.75-1.36 (m, $\left.24 \mathrm{H}\right), 3.24-3.32(\mathrm{~m}, 4 \mathrm{H}), 5.03$ $(\mathrm{s}, 4 \mathrm{H}), 7.12-7.39(\mathrm{~m}, 14 \mathrm{H}), 7.92-7.97(\mathrm{~m}, 6 \mathrm{H}), 8.23(\mathrm{~s}, 2 \mathrm{H}) ;{ }^{13} \mathrm{C}$ NMR $\left(100 \mathrm{MHz}, \mathrm{CD}_{2} \mathrm{Cl}_{2}\right) \delta=$ 24.4, 24.5, 24.6, 24.7, 45.2, 45.3, 69.1, 69.2, 122.6, 124.7, 125.1, 126.1, 126.2, 127.0, 127.3, 128.6, 128.8, 129.1, 130.0, 131.0, 131.2, 131.4, 132.6, 132.8, 133.1, 148.7, 150.9, 167.9; ${ }^{31} \mathrm{P}$ NMR (161.7 $\mathrm{MHz}, \mathrm{CD}_{2} \mathrm{Cl}_{2}$ ) $\delta=150.7$; MS (m/z) 43 (31), 57 (32), 71 (26), 149 (100), 266 (27), 281 (49), 329 (87), 391 (28), 444 (50), 772 (18), $873\left(43,[\mathrm{M}+\mathrm{H}]^{+}\right)$; exact mass calcd for $\mathrm{C}_{54} \mathrm{H}_{55} \mathrm{~N}_{2} \mathrm{O}_{5} \mathrm{P}_{2}\left([\mathrm{M}+\mathrm{H}]^{+}\right)$: 873.3586; found 873.3604.

\subsection{Rhodium complexes (7, Scheme 2) \\ 4.3.1. Complex between $\left[R h(n b d)_{2}\right] B_{4}$ and $5 a(7 a)$}

To a solution of $5 \mathbf{a}(0.05 \mathrm{mmol})$ in $\mathrm{CD}_{2} \mathrm{Cl}_{2}$ was added $\left[\mathrm{Rh}(\mathrm{nbd})_{2}\right] \mathrm{BF}_{4}(0.05 \mathrm{mmol})$ under atmosphere of argon. The solvent was evaporated to dryness in vacuo to give solids of 7a. All attempts at synthesizing single crystals were failed. ${ }^{31} \mathrm{P}$ NMR $\left(161.7 \mathrm{MHz}, \mathrm{CD}_{2} \mathrm{Cl}_{2}\right) \delta=142.4(\mathrm{~d}$, $J_{\mathrm{Rh}-\mathrm{P}}=248.9 \mathrm{~Hz}$ ); exact mass calcd for $\mathrm{C}_{53} \mathrm{H}_{46} \mathrm{BF}_{4} \mathrm{~N}_{2} \mathrm{O}_{5} \mathrm{P}_{2} \mathrm{Rh}$ ([M+1$\left.\left.-\mathrm{BF}_{4}\right]\right)$ : 955.1938; found 955.1913

\subsubsection{Complex between $\left[\mathrm{Rh}(\mathrm{COe})_{2} \mathrm{Cl}\right]_{2}$ and $5 \boldsymbol{a}(\mathbf{7 b})$}

${ }^{31} \mathrm{P}$ NMR $(161.7 \mathrm{MHz})$ of a mixture between $5 \mathbf{a}(0.05 \mathrm{mmol})$ and $\left[\mathrm{RhCl}(\mathrm{coe})_{2}\right]_{2}(0.025$ $\mathrm{mmol})$ in $\mathrm{CD}_{2} \mathrm{Cl}_{2}$ exhibited a signal at $\delta=153.7\left(\mathrm{~d}, J_{\mathrm{Rh}-\mathrm{P}}=296.3 \mathrm{~Hz}\right)$. 


\subsubsection{Complex between $\left[R h(n b d)_{2}\right] B F_{4}$ and $5 \boldsymbol{b}$}

${ }^{31} \mathrm{P}$ NMR $(161.7 \mathrm{MHz})$ of a mixture between $5 \mathbf{b}(0.05 \mathrm{mmol})$ and $\left[\mathrm{Rh}(\mathrm{nbd})_{2}\right] \mathrm{BF}_{4}(0.05$ mmol) in $\mathrm{CD}_{2} \mathrm{Cl}_{2}$ exhibited a signal at $\delta=142.3\left(\mathrm{~d}, J_{\mathrm{Rh}-\mathrm{P}}=248.9 \mathrm{~Hz}\right)$.

\subsubsection{Complex between $\left[R h(n b d)_{2}\right] B F_{4}$ and $5 c$}

${ }^{31} \mathrm{P}$ NMR $(161.7 \mathrm{MHz})$ of a mixture between $5 \mathrm{c}(0.05 \mathrm{mmol})$ and $\left[\mathrm{Rh}(\mathrm{nbd})_{2}\right] \mathrm{BF}_{4}(0.05$ $\mathrm{mmol}$ ) in $\mathrm{CD}_{2} \mathrm{Cl}_{2}$ exhibited three signals at $\delta=24.8,111.0,134.1$.

\subsection{General procedure for asymmetric 1,4-addition (Table 1)}

A flask charged with $\left[\mathrm{Rh}(\mathrm{coe}){ }_{2} \mathrm{Cl}\right]_{2}(0.015 \mathrm{mmol}), 4\left(\mathrm{R}^{1}, \mathrm{R}^{2}=\mathrm{Et}\right)(0.066 \mathrm{mmol})$ was flushed with argon. 1,3-Dioxane $(2.6 \mathrm{ml})$ was then added. After being stirred for $1 \mathrm{~h}$ at room temperature, arylboronic acid $(1.5 \mathrm{mmol})$, enone $(1.0 \mathrm{mmol})$, and aqueous $\mathrm{KOH}(2.4 \mathrm{M}, 0.43 \mathrm{ml}, 1 \mathrm{mmol})$ were added. The resulting mixture was stirred for $6 \mathrm{~h}$ at $50{ }^{\circ} \mathrm{C}$. Isolated yields determined by chromatography on silica gel are shown in Table 1. Enantiomer excess was determined by HPLC analyses using a chiral stationary column (Dicel Chiralpak AD and Chiralcel OD-H or OB-H).

We previously reported the spectral data of 3a [5l], 3b [5l], 3c [5l], 3d [5a], 3e [5l], 3f [4f], $3 \mathbf{g}[5 \mathrm{a}]$, and $\mathbf{3 h}$ [5l]. The specific rotations of these compounds were $3 \mathbf{a}\left([\alpha]_{\mathrm{D}}{ }^{21}=+20.4^{\circ}(\mathrm{c}=1.03\right.$, $\left.\left.\mathrm{CHCl}_{3}\right)\right)$, $3 \mathbf{b}\left([\alpha]_{\mathrm{D}}{ }^{22}=+13.6^{\circ}\left(\mathrm{c}=0.98, \mathrm{CHCl}_{3}\right)\right), \mathbf{3 c}\left([\alpha]_{\mathrm{D}}{ }^{22}=+17.3^{\circ}\left(\mathrm{c}=0.96, \mathrm{CHCl}_{3}\right)\right), \mathbf{3 d}\left([\alpha]_{\mathrm{D}}{ }^{21}=\right.$ $\left.+79.9^{\circ}\left(\mathrm{c}=1.13, \mathrm{CHCl}_{3}\right)\right), 3 \mathbf{e}\left([\alpha]_{\mathrm{D}}^{21}=+71.4^{\circ}\left(\mathrm{c}=0.92, \mathrm{CHCl}_{3}\right)\right), 3 \mathbf{f}\left([\alpha]_{\mathrm{D}}^{22}=+58.8^{\circ}(\mathrm{c}=1.07\right.$, $\left.\left.\mathrm{CHCl}_{3}\right)\right), 3 \mathbf{g}\left([\alpha]_{\mathrm{D}}{ }^{23}=+59.3^{\circ}\left(\mathrm{c}=1.00, \mathrm{CHCl}_{3}\right)\right)$, and $3 \mathbf{h}\left([\alpha]_{\mathrm{D}}{ }^{21}=+16.1^{\circ}\left(\mathrm{c}=0.97, \mathrm{CHCl}_{3}\right)\right)$.

\subsection{General procedure for asymmetric 1,4-addition (Table 3 and Scheme 3)}

A flask charged with $\left[\mathrm{Rh}(\mathrm{nbd})_{2}\right]_{\mathrm{BF}_{4}}(0.03 \mathrm{mmol}, 3 \mathrm{~mol} \%)$ and $5 \mathrm{a}(0.033 \mathrm{mmol})$ was flushed with argon. 1,3-Dioxane $(2.6 \mathrm{ml})$ and water $(0.43 \mathrm{ml})$ were then added. After being stirred for $0.5 \mathrm{~h}$, arylboronic acid (1.5 mmol), $\alpha, \beta$-unsaturated carbonyl compound $(1.0 \mathrm{mmol})$ and triethylamine $(1 \mathrm{mmol})$ were successively added. The resulting mixture was stirred at $5{ }^{\circ} \mathrm{C}$ or $25{ }^{\circ} \mathrm{C}$. Isolated yields determined by chromatography on silica gel are shown in Table 2. Enantiomer excess was determined by HPLC analyses using a chiral stationary column (Dicel Chiralpak AD and Chiralcel OD-H or OB-H).

\subsubsection{4-(3-Fluorophenyl)nonan-2-one (3m)}

$[\alpha]_{\mathrm{D}}{ }^{21}=+14.8^{\circ}\left(\mathrm{c}=1.00, \mathrm{CHCl}_{3}\right) ;{ }^{1} \mathrm{H} \mathrm{NMR}\left(400 \mathrm{MHz}, \mathrm{CDCl}_{3}\right) \quad \delta \quad 0.74-0.77$ (m, 3H), 1.02-1.18 (m, 6H), 1.43-1.54 (m, 2H), 1.96 (s, 3H), 2.63 (d, J=7.3 Hz, 2H), 3.02-3.09 (m, 1H), 
6.78-6.89 (m, 3H), 7.13-7.19 (m, 2H); ${ }^{13} \mathrm{C}$ NMR (100 MHz, $\left.\mathrm{CDCl}_{3}\right) \quad \delta \quad$ 14.0, 22.4, 26.9, 30.6, 31.6, 36.2, 40.9, 50.6, 113.1 (d, $J=20.7 \mathrm{~Hz}$ ), 114.1 (d, $J=21.5 \mathrm{~Hz}$ ), 123.3 (d, $J=2.5 \mathrm{~Hz}$ ), 129.8 (d, $J=8.3$ Hz), 147.4, 162.9 (d, J=245.6 Hz), 207.4; MS (m/z) 43 (54), 55 (9), 109 (43), 122 (64), 135 (19), 165 (63), 178 (100), 236 (11, $\mathrm{M}^{+}$); exact mass calcd for $\mathrm{C}_{15} \mathrm{H}_{21} \mathrm{FO}$ : 236.1576; found 236.1575.

\subsubsection{3-(4-Methoxyphenyl)-1-phenyloctan-1-one (3n)}

$[\alpha]_{\mathrm{D}}{ }^{22}=-2.9^{\circ}\left(\mathrm{c}=0.97, \mathrm{CHCl}_{3}\right) ;{ }^{1} \mathrm{H} \mathrm{NMR}\left(400 \mathrm{MHz}, \mathrm{CDCl}_{3}\right) \quad \delta \quad 0.81-0.84(\mathrm{~m}, 3 \mathrm{H})$, 1.21-1.27 (m, 6H), 1.59-1.73 (m, 2H), 3.17-3.34 (m, 3H), 3.78 (s, 3H), 6.71-6.84 (m, 3H), 7.18-7.55 (m, 4H), 7.89-7.91 (m, 2H); ${ }^{13} \mathrm{C}$ NMR (100 MHz, $\left.\mathrm{CDCl}_{3}\right) \quad \delta$ 14.06, 22.51, 27.17, 31.78, 36.20, 41.31, 45.93, 55.11, 111.14, 113.58, 119.98, 128.1, 128.5, 129.35, 132.90, 137.23, 146.77, 159.58, 199.15 ; MS (m/z) 55 (13), 77 (44), 105 (75), 121 (11), 135 (27), 190 (100), 205 (33), 239 (49), 310 (29, $\mathrm{M}^{+}$); exact mass calcd for $\mathrm{C}_{21} \mathrm{H}_{26} \mathrm{O}_{2}$ : 310.1933; found 310.1931

\subsubsection{5-Methyl-3-(3-fluorophenyl)hexan-2-one (3q)}

$[\alpha]_{\mathrm{D}}{ }^{21}=+25.7^{\circ}\left(\mathrm{c}=0.95, \mathrm{CHCl}_{3}\right) ;{ }^{1} \mathrm{H} \mathrm{NMR}\left(400 \mathrm{MHz}, \mathrm{CDCl}_{3}\right) \quad \delta \quad 0.67(\mathrm{~d}, J=6.95 \mathrm{~Hz}, 3 \mathrm{H})$, 0.86 (d, J=6.95 Hz, 3H), 1.69-1.78 (m, 1H), 1.93 (s, 3H), 2.65-2.77 (m, 2H), 2.83-2.89 (m, 1H), 6.76-6.86 (m, 3H), 7.12-7.19 (m, 2H); ${ }^{13} \mathrm{C}$ NMR (100 MHz, $\left.\mathrm{CDCl}_{3}\right) \quad \delta \quad$ 20.3, 20.6, 30.6, 33.2, 47.4, 47.6, 113.1 (d, $J=21.5 \mathrm{~Hz}$ ), 114.9 (d, $J=21.5 \mathrm{~Hz}$ ), 124.0 (d, $J=3.3 \mathrm{~Hz}), 129.5$ (d, $J=8.3 \mathrm{~Hz}), 146.1$ (d, $J=6.6$ Hz), 162.7 (d, J=245.7 Hz), 207.7; MS (m/z) 43 (70), 109 (23), 123 (32), 135 (11), 150 (100), 166 (15), 208 (4, $\left.\mathrm{M}^{+}\right)$; exact mass calcd for $\mathrm{C}_{13} \mathrm{H}_{17} \mathrm{FO}$ : 208.1263; found 208.1264.

\subsubsection{1-Cyclohexyl-4-methyl-3-(3-methoxyphenyl)pentan-1-one (3r)}

$[\alpha]_{\mathrm{D}}{ }^{22}=+54.5^{\circ}\left(\mathrm{c}=0.50, \mathrm{CHCl}_{3}\right) ;{ }^{1} \mathrm{H} \mathrm{NMR}\left(400 \mathrm{MHz}, \mathrm{CDCl}_{3}\right) \quad \delta \quad 0.74(\mathrm{~d}, J=6.8 \mathrm{~Hz}, 3 \mathrm{H})$, 0.92 (d, $J=6.6$ Hz, 3H), 1.06-1.29 (m, 4H), 1.56-1.85 (m, 7H), 2.19-2.21 (m, 1H), 2.79 (d, J=7.3 Hz, 2H), 2.93 (dt, J=7.3 Hz, 7.1 Hz, 1H), 3.79 (s, 3H), 6.68-6.74 (m, 3H), 7.15-7.26 (m, $1 \mathrm{H}) ;{ }^{13} \mathrm{C}$ NMR $\left(100 \mathrm{MHz}, \mathrm{CDCl}_{3}\right) \quad \delta \quad 20.4,20.8,25.59,25.61,25.8,28.1,28.2,33.0,44.8,47.5,51.2,55.1,111.0$, 114.3, 120.8, 128.9, 145.6, 159.3, 213.1; MS (m/z) 55 (22), 83 (51), 121 (30), 162 (100), 177 (17), $288\left(16, \mathrm{M}^{+}\right.$); exact mass calcd for $\mathrm{C}_{19} \mathrm{H}_{28} \mathrm{O}_{2}$ : 288.2089; found 288.2099.

\subsubsection{4-Methyl-3-(3-methoxyphenyl)-1-phenylpentan-1-one (3s)}

$[\alpha]_{\mathrm{D}}{ }^{21}=-1.8^{\circ}\left(\mathrm{c}=0.92, \mathrm{CHCl}_{3}\right) ;{ }^{1} \mathrm{H} \mathrm{NMR}\left(400 \mathrm{MHz}, \mathrm{CDCl}_{3}\right) \quad \delta \quad 0.800(\mathrm{~d}, J=6.6 \mathrm{~Hz}, 3 \mathrm{H})$, 0.98 (d, $J=6.8 \mathrm{~Hz}, 3 \mathrm{H}), 1.86-1.97$ (m, 1H), 3.11-3.17 (dd, $J=7.2,14.3 \mathrm{~Hz}, 1 \mathrm{H}), 3.34$ (d, J=6.8 Hz, 2H), 3.76 (s, 3H), 6.68-6.79 (m, 3H), 7.16 (t, J=7.8 Hz, 1H), 7.39-7.43 (m, 2H), 7.50-7.54 (m, 1H), 
7.84-7.94 (m, 2H); ${ }^{13} \mathrm{C}$ NMR (100 MHz, $\left.\mathrm{CDCl}_{3}\right) \quad \delta \quad 20.5,20.9,33.3,42.5,47.8,55.1,111.1,114.4$, 120.8, 128.0, 128.5, 129.0, 132.8, 137.3, 145.4, 159.3, 199.4; MS (m/z) 77 (35), 105 (100), 162 (91), 177 (8), 239 (8), $282\left(10, \mathrm{M}^{+}\right)$; exact mass calcd for $\mathrm{C}_{19} \mathrm{H}_{22} \mathrm{O}_{2}$ : 282.1620; found 282.1623.

\subsubsection{Methyl-3-(3-methoxyphenyl)butanoate (3v)}

$[\alpha]^{21}{ }_{\mathrm{D}}=+23.0^{\circ}\left(\mathrm{c}=0.56, \mathrm{CHCl}_{3}\right) ;{ }^{1} \mathrm{H} \mathrm{NMR}\left(400 \mathrm{MHz}, \mathrm{CDCl}_{3}\right) \quad \delta \quad 1.29(\mathrm{~d}, J=7.1 \mathrm{~Hz}, 3 \mathrm{H})$, 2.56 (dd, $J=8.3,15.2$ Hz, 1H), 2.63 (dd, J=6.6, 15.3 Hz, 1H), 3.21-3.30 (m, 1H), 3.63 (s, 3H), 3.80 (s, 3H), 6.74-6.83 (m, 3H), 7.20-7.26 (m, 2H); ${ }^{13} \mathrm{C}$ NMR (100 MHz, $\left.\mathrm{CDCl}_{3}\right) \delta \quad$ 21.7, 36.4, 42.6, 51.5, 55.1, 111.4, 112.7, 119.0, 129.5, 147.4, 159.6, 172.8; MS (m/z) 77 (10), 91 (13), 105 (26), 121 (11), 135 (84), 148 (100), 208 (57, $\mathrm{M}^{+}$); exact mass calcd for $\mathrm{C}_{12} \mathrm{H}_{16} \mathrm{O}_{3}$ : 208.1099; found 208.1091.

\subsubsection{4-Phenyldihydrofuran-2-one (3w)}

$[\alpha]_{\mathrm{D}}{ }^{22}=-39.2^{\circ}\left(\mathrm{c}=0.99, \mathrm{CHCl}_{3}\right) ;{ }^{1} \mathrm{H} \mathrm{NMR}\left(400 \mathrm{MHz}, \mathrm{CDCl}_{3}\right) \quad \delta 2.60$ (dd, $J=9.3,17.6 \mathrm{~Hz}$, 1H), 2.85 (dd, $J=8.8,8.8 \mathrm{~Hz}, 1 \mathrm{H}$ ), 3.68-3.76 (m, 1H), 4.60 (dd, $J=7.8,9.02 \mathrm{~Hz}, 1 \mathrm{H}$ ), 4.20 (dd, $J=7.8$, $8.8 \mathrm{~Hz}, 1 \mathrm{H}), 7.15-7.32$ (m, 5H); ${ }^{13} \mathrm{C} \mathrm{NMR}\left(100 \mathrm{MHz}, \mathrm{CDCl}_{3}\right) \quad \delta \quad 35.7,41.0,74.0,126.7,127.7$, 129.1, 139.4, 176.4; MS (m/z) 51 (12), 78 (12), 104 (100), 162 (23, $\mathrm{M}^{+}$); exact mass calcd for $\mathrm{C}_{10} \mathrm{H}_{10} \mathrm{O}_{2}$ : 162.0681; found 162.0695 .

\subsubsection{4-Phenyltetrahydropyran-2-one (3x)}

$[\alpha]_{\mathrm{D}}{ }^{22}=-2.8^{\circ}\left(\mathrm{c}=1.01, \mathrm{CHCl}_{3}\right) ;{ }^{1} \mathrm{H} \mathrm{NMR}\left(400 \mathrm{MHz}, \mathrm{CDCl}_{3}\right) \delta 1.91-2.01(\mathrm{~m}, 1 \mathrm{H})$, 2.07-2.14 (m, 1H), 2.56 (dd, $J=10.7,17.6 \mathrm{~Hz}, 1 \mathrm{H}$ ), 3.15 (ddd, $J=17.6,5.9,1.7 \mathrm{~Hz}, 1 \mathrm{H}$ ), 3.12-3.20 (m, 1H), 4.31 (ddd, $J=11.8,10.9,3.9 \mathrm{~Hz}, 1 \mathrm{H}$ ), 4.43 (ddd, $J=11.5,5.0,3.9 \mathrm{~Hz}, 1 \mathrm{H}$ ), 7.13-7.22 (m, 3H), 7.26-7.30 (m, 2H). ${ }^{13} \mathrm{C}$ NMR (100 MHz, $\left.\mathrm{CDCl}_{3}\right) \quad \delta \quad 30.2$, 37.3, 47.4, 68.6, 126.4, 127.1, 128.9, 142.7, 170.6; MS (m/z) 51 (9), 78 (20), 92 (26), 104 (65), 117 (87), 130 (16), 158 (16), 176 $\left(100, \mathrm{M}^{+}\right)$; exact mass calcd for $\mathrm{C}_{12} \mathrm{H}_{14} \mathrm{O}_{2}$ 176.0837; found 176.0836.

\subsubsection{4-(3-Methoxyphenyl)tetrahydropyran-2-one (3y)}

$[\alpha]_{\mathrm{D}}{ }^{22}=+4.0^{\circ}\left(\mathrm{c}=0.51, \mathrm{CHCl}_{3}\right) ;{ }^{1} \mathrm{H}$ NMR $\left(400 \mathrm{MHz}, \mathrm{CDCl}_{3}\right) \delta 1.98-2.08(\mathrm{~m}, 1 \mathrm{H}), 2.14-2.21(\mathrm{~m}$, 1H), 2.63 (dd, $J=10.7,17.6 \mathrm{~Hz}, 1 \mathrm{H}$ ), 2.92 (ddd, $J=17.7,5.85,1.46 \mathrm{~Hz}, 1 \mathrm{H}$ ), 3.17-3.25 (m, 1H), 4.38 (ddd, $J=11.0,11.0,3.7 \mathrm{~Hz}, 1 \mathrm{H}$ ), 4.50 (ddd, $J=11.5$, 4.6, 3.9 Hz, 1H), 6.74-6.83 (m, 3H), 7.14-7.36 (m, 1H). $\left.{ }^{13} \mathrm{C} \mathrm{NMR} \mathrm{(100} \mathrm{MHz,} \mathrm{CDCl}_{3}\right) \quad \delta \quad 30.2,37.4,55.2,68.6,112.1,112.7,118.6,130.0,144.4$, 160.0, 170. 6; MS (m/z) 65 (18), 77 (19), 91 (35), 121 (38), 134 (86), 150 (31), 163 (25), 206 (100, $\mathrm{M}^{+}$); exact mass calcd for $\mathrm{C}_{12} \mathrm{H}_{14} \mathrm{O}_{3}$ : 206.0943; found 206.0937 . 
The spectral data of $\mathbf{3 i}$ [5l], 3j [5l], 3k [5b], 31 [5l], 3o [5l], 3p [5l], 3t [4f] and 3u [4f] were reported previously. The specific rotations of these compounds were $3 \mathbf{i}\left([\alpha]_{\mathrm{D}}{ }^{22}=+17.5^{\circ}(\mathrm{c}=0.97\right.$, $\left.\left.\mathrm{CHCl}_{3}\right)\right), 3 \mathbf{j}\left([\alpha]_{\mathrm{D}}{ }^{21}=+16.4^{\circ}\left(\mathrm{c}=0.93, \mathrm{CHCl}_{3}\right)\right), 3 \mathbf{k}\left([\alpha]_{\mathrm{D}}{ }^{22}=+70.8^{\circ}\left(\mathrm{c}=1.02, \mathrm{CHCl}_{3}\right)\right), 3 \mathbf{l}\left([\alpha]_{\mathrm{D}}{ }^{23}=\right.$ $\left.+15.9^{\circ}\left(\mathrm{c}=0.93, \mathrm{CHCl}_{3}\right)\right), 3 \mathbf{0}\left([\alpha]_{\mathrm{D}}{ }^{21}=+32.4^{\circ}\left(\mathrm{c}=0.98, \mathrm{CHCl}_{3}\right)\right), 3 \mathbf{p}\left([\alpha]_{\mathrm{D}}{ }^{21}=+18.2^{\circ}(\mathrm{c}=0.51\right.$, $\left.\left.\mathrm{CHCl}_{3}\right)\right), 3 \mathbf{t}\left([\alpha]_{\mathrm{D}}{ }^{23}=-1.6^{\circ}\left(\mathrm{c}=0.51, \mathrm{CHCl}_{3}\right)\right)$ and $3 \mathbf{u}\left([\alpha]_{\mathrm{D}}{ }^{22}=-4.4^{\circ}\left(\mathrm{c}=0.91, \mathrm{CHCl}_{3}\right)\right)$.

\section{Acknowledgments}

This work was supported by Grant-in-Aid for Scientific Research on Priority Areas (No. 14078101, "Reaction Control of Dynamic Complexes") from Ministry of Education, Culture, Sports, Science and Technology, Japan.

\section{References}

[1] For reviews, see: (a) N. Krause, A. Hoffmann-Röder, Synthesis (2001) 171;

(b) K. Tomioka, Y. Nagaoka in: E. N. Jacobsen, A. Pfalts, H. Yamamoto (Eds), Comprehensive Asymmetric Catalysis, Springer-Verlag, Berlin, 1999, chapter 31.1.

[2] (a) A. Alexakis, C. Benhaim, Eur. J. Org. Chem. (2002) 3221;

(b) B. L. Feringa, Acc. Chem. Res. 33 (2000) 346.

[3] For reviews, see: (a) T. Hayashi, K. Yamasaki, Chem. Rev. 103 (2003) 2829;

(b) K. Fagnou, M. Lautens, Chem. Rev. 103 (2000) 169.

[4] (a) T. Nishikata, Y. Yamamoto, N. Miyaura, Angew. Chem., Int. Ed. 42 (2003) 2768;

(b) T. Nishikata, Y. Yamamoto, N. Miyaura, Chem. Lett. 32 (2003) 752;

(c) T. Nishikata, Y. Yamamoto, N. Miyaura, Chem. Commun. (2004) 1822;

(d) T. Nishikata, Y. Yamamoto, N. Miyaura, Organometallics 23 (2004) 4317;

(e) T. Nishikata, Y. Yamamoto, N. Miyaura, Chem. Lett. 34 (2005) 721;

(f) T. Nishikata, Y. Yamamoto, I. D. Gridnev, N. Miyaura, Organometallics, 24 (2005) 5025.

[5] (a) Y. Takaya, M. Ogasawara, T. Hayashi, M. Sakai, N. Miyaura, J. Am. Chem. Soc. 120 (1998) 5579;

(b) Y. Takaya, M. Ogasawara, T. Hayashi, Tetrahedron Lett. 40 (1999) 6957;

(c) T. Hayashi, T. Senda, Y. Takaya, M. Ogasawara, J. Am. Chem. Soc. 121 (1999) 11591;

(d) T. Hayashi, T. Senda, M. Ogasawara, J. Am. Chem. Soc. 122 (2000) 10716; 
(e) S. Sakuma, M. Sakai, R. Itooka, N. Miyaura, J. Org. Chem. 65 (2000) 5951;

(f) S. Sakuma, N. Miyaura, J. Org. Chem. 66 (2001) 8944;

(g) K. Yoshida, M. Ogasawara, T. Hayashi, J. Am. Chem. Soc. 124 (2002) 10984;

(h) T. Hayashi, M. Takahashi, Y. Tkaya M. Ogasawara, J. Am. Chem. Soc. 124 (2002) 5052;

(i) M. Pucheault, S. Darses, J.-P. Genet, Tetrahedron Lett. 43 (2002) 6157;

(j) T. Hayashi, K. Ueyama, N. Tokunaga and K. Yoshida, J. Am. Chem. Soc. 125 (2003) 11508;

(k) D. F. Cauble, J. D. Gipson, M. J. Krische, J. Am. Chem. Soc. 125 (2003) 1110;

(l) R. Itooka, Y. Iguchi, N. Miyaura, J. Org. Chem. 68 (2003) 6000.

[6] (a) Y. Iguchi, R. Itooka, N, Miyaura, Synlett (2003) 1040;

(b) J,-G, Boiteau, R. Imbos, A. J. Minnard, B. L. Feringa, Org. Lett. 5 (2003) 681;

(c) A. Duursma, R. Hoen, J. Schuppan, R. Hulst, A. J. Minnard, B. L. Feringa, Org. Lett. 5 (2003) 3111;

(d) J.-G. Boiteau, A. J. Minnard, B. L. Feringa, J. Org. Chem. 68 (2003) 9481;

(e) A. Duursma, J. -G. Boiteau, L. Lefort, J. A. F. Boorgers, A. H. M. de Vries, J. G. de Vries, A. J. Minnard, B. L. Feringa, J. Org. Chem. 69 (2004) 8045;

(f) S. L. X. Martina, A. J. Minnaard, B. Hessen, B. L. Feringa, Tetrahedron Lett. 46 (2005) 7159 .

[7] P. Mauleon, J. C. Carretero, Org. Lett. 6 (2004) 3195.

[8] M. T. Reetz, D. Moulin, A. Gosberg, Org. Lett. 3 (2001) 4083.

[9] M. Kuriyama, K. Nagai, K. Yamada, Y. Miwa, T. Taga, K. Tomioka, J. Am. Chem. Soc. 124 (2002) 8932.

[10] (a) T. Hayashi, K. Ueyama, N. Tokunaga, K. Yoshida, J. Am. Chem. Soc. 125 (2003) 11508;

(b) N. Tokunaga, Y. Otomaru, K. Okamoto, K. Ueyama, R. Shintani, T. Hayashi, J. Am. Chem. Soc. 126 (2004) 13584;

(c) C. Defieber, J.-F. Paquin, S. Serna, E. M. Carreira, Org. Lett. 6 (2004) 3873;

(d) Y. Otomaru, K. Okamoto, R. Shintani, T. Hayashi, J. Org. Chem. 70 (2005) 2503.

(e) F. -X. Chen, A. Kina, T. Hayashi, Org. Lett. 8 (2006) 341.

[11] Y. Ma, C. Song, C. Ma, Z. Sun, Q. Chai, M. B. Andrus, Angew. Chem., Int. Ed. 42 (2003) 5871.

[12] (a) A. H. M. de Vries, A. Meetsma, B. L. Feringa, Angw. Chem., Int. Ed. 35 (1996) 2374;

(b) B. L. Feringa, M. Pineschi, L. A. Arnold, R. Imbos, A. H. M. de Vries, Angew. Chem., Int. Ed. 36 (1997) 2620; 
(c) L. A. Arnold, R. Imbos, A. Mandoli, A. H. M. de Vries, R. Naasz, B. L. Feringa, Tetrahedron 56 (2000) 2865.

[13] Y. Yamamoto, K. Kurihara, N. Sugishita, K. Oshita, D. Piao, N. Miyaura, Chem. Lett. 34 (2005) 1224.

[14] (a) M. Bougauchi, A. Watanabe, T. Arai, H. Sasai, M. Shibasaki, J. Am. Chem. Soc. 119 (1997) 2329;

(b) S. Matsunaga, J. Das, J. Roels, E. M. Vogl, N. Yamamoto, T. Iida, K. Yamaguchi, M. Shibasaki, J. Am. Chem. Soc. 122 (2000) 2252;

(c) N. Kumagai, S. Matsunaga, T. Kinoshita, S. Harada, S. Okada, S. Sakamoto, K. Yamaguchi, M. Shibasaki, J. Am. Chem. Soc. 125 (2003) 2169;

(d) K. Majima, R. Takita, A. Okada, T. Oshima, M. Shibasaki, J. Am. Chem. Soc. 125 (2003) 15837.

[15] R. Hulst, N. K. de Vries, B. L. Feringa, Tetrahedron: Asymmetry 5 (1996) 699.

[16] A. van der Ent, A. L. Onderdelinden, Inorg. Synth. 14 (1973) 93.

[17] T. G. Schenck, J. M. Downes, C. R. C. Milne, P. B. Mackenzie, H. Boucher, J. Whelan, B. Bosnich, Inorg. Chem. 24 (1985) 2334. 


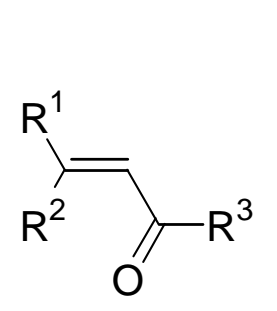

1

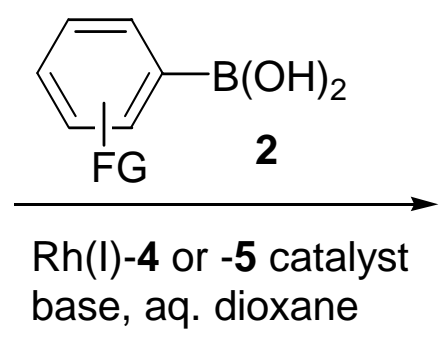

base, aq. dioxane

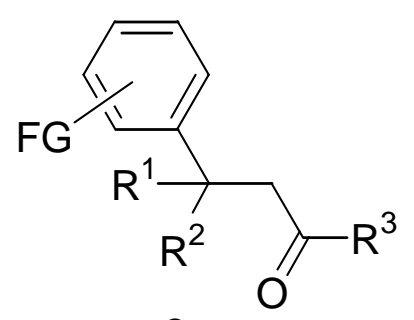

3<smiles>[R]N([R])p1oc2ccc3ccccc3c2c2c(ccc3ccccc32)o1</smiles>

4<smiles>[R]N([R])P1Oc2ccccc2-c2c(c3cc(COCC4=Cc5ccccc5C5=c6ccccc6=CC=C4O5)c2op(N([R])[R])o3)-c2c(ccc3ccccc23)O1</smiles>

5a: $\mathrm{R}=\mathrm{Me}$

5b: $\mathrm{R}=\mathrm{Et}$

5c: $\mathrm{R}=i-\mathrm{Pr}$

Scheme 1. Asymmetric 1,4-addition of arylboronic acids to enones catalyzed by $\mathrm{Rh}(\mathrm{I})$-phosphoramidite complexes 

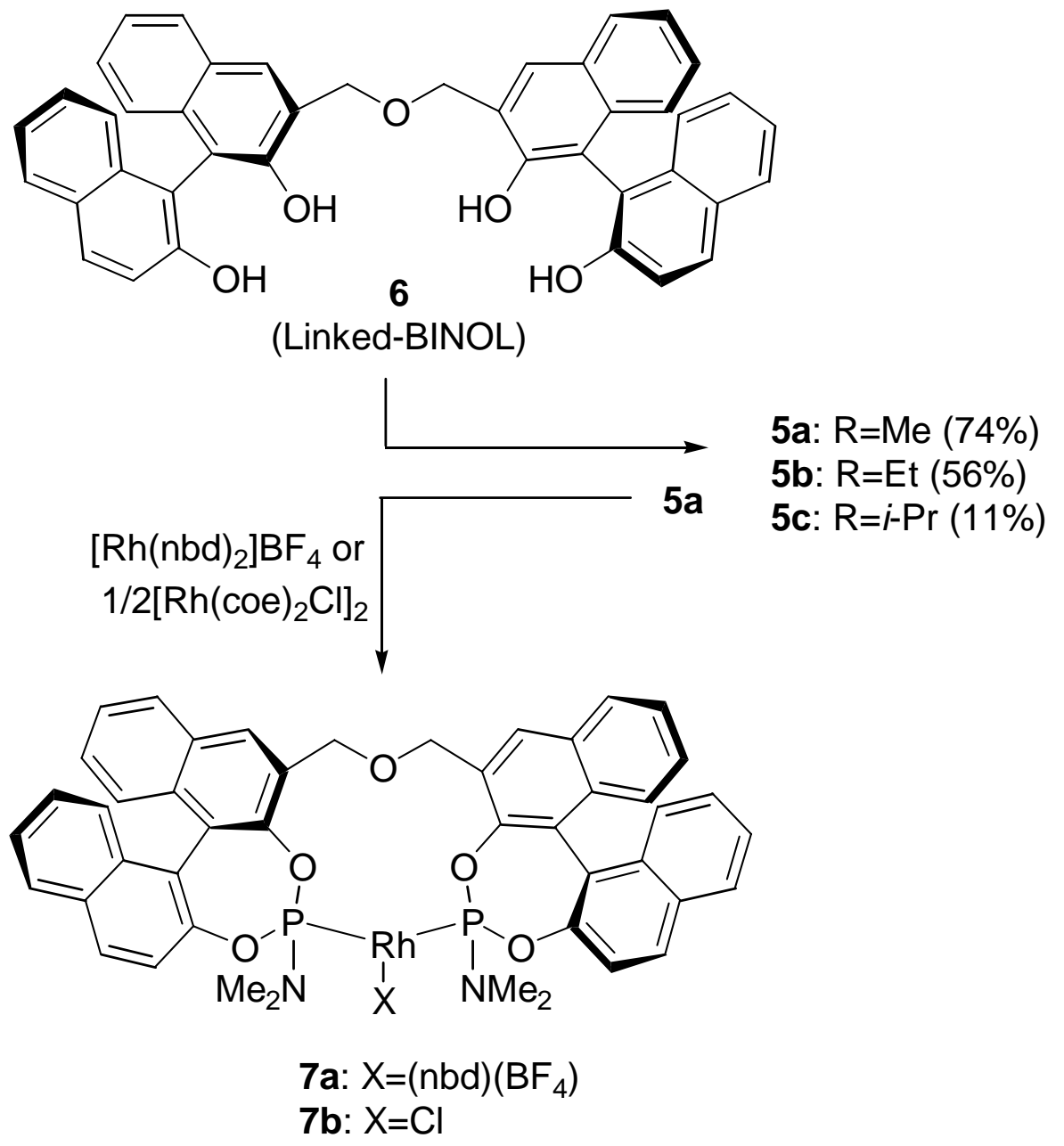

Scheme 2. Phosphoramidites based on Linked-BINOL 


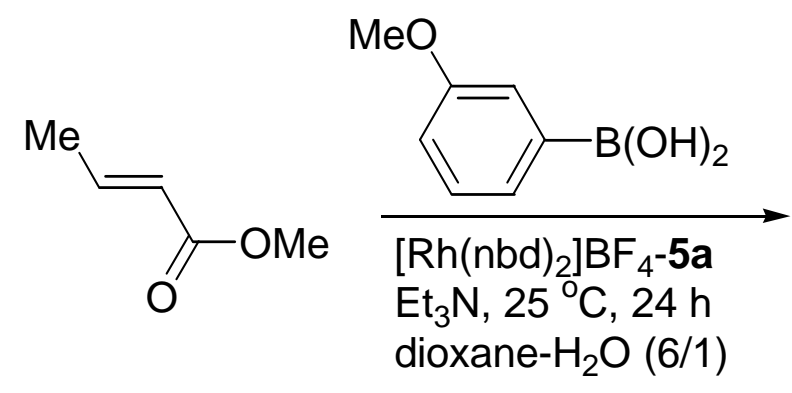

dioxane- $\mathrm{H}_{2} \mathrm{O}(6 / 1)$

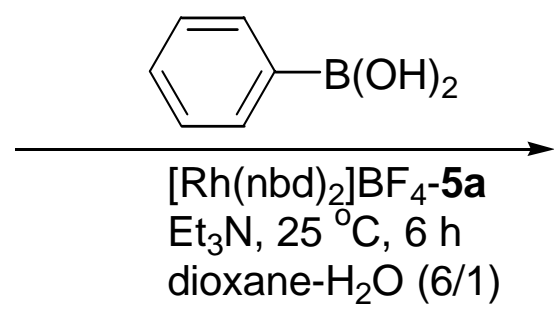

$\underbrace{11}$

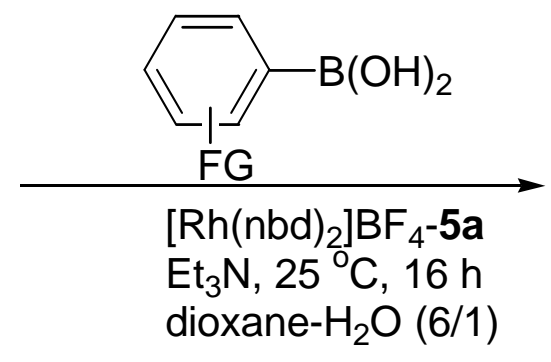

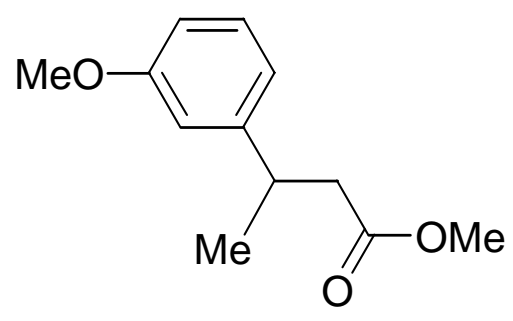

(1)<smiles>O=C1CC(c2ccccc2)CO1</smiles>

$3 w, 68 \%, 77 \%$ ee<smiles>O=C1CC(c2ccc(F)cc2)CCO1</smiles>

3x: $F G=H, 72 \%, 89 \%$ ee

3y: FG=3-MeO, 61\%, 91\%ee

Scheme 3. Asymmetric 1,4-addition to unsaturated esters 


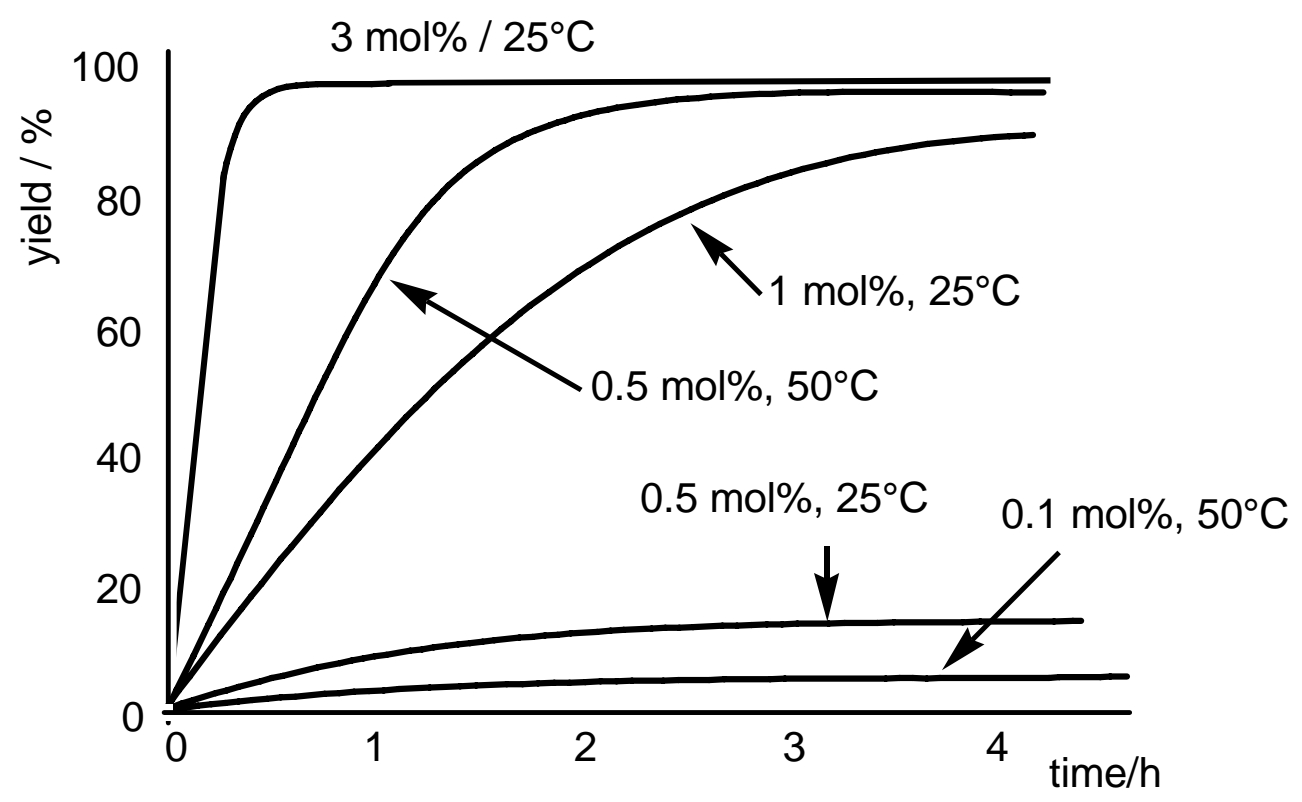

Figure 1. Amounts of catalyst loading and reaction rates 
Table 1. 1,4-Addition of arylboronic acid to $\alpha, \beta$-unsaturated carbonyl compounds catalyzed by Rh(I)-4 complexes ${ }^{[a]}$

\begin{tabular}{|c|c|c|c|c|c|c|c|}
\hline \multirow[t]{2}{*}{ entry } & \multirow[t]{2}{*}{ carbonyl compound } & \multicolumn{2}{|c|}{ ligand (1) } & \multicolumn{2}{|c|}{$\operatorname{ArB}(\mathrm{OH})_{2}$, product } & \multirow[t]{2}{*}{ yield/\% ${ }^{[\mathrm{b}]}$} & \multirow[t]{2}{*}{$\% \mathrm{ee}^{[\mathrm{c}]}$} \\
\hline & & $\mathrm{R}^{1}=$ & $\mathrm{R}^{2}=$ & $X=$ & No & & \\
\hline 1 & 2-cyclohexenone & $\mathrm{Me}$ & $\mathrm{Me}$ & $\mathrm{H}$ & 3a & 85 & $85(R)$ \\
\hline 2 & 2-cyclohexenone & Et & Et & $\mathrm{H}$ & 3a & 89 & $91(R)$ \\
\hline 3 & 2-cyclohexenone & Et & Et & 3-MeO & $3 \mathbf{b}$ & 85 & 98 \\
\hline 4 & 2-cyclohexenone & Et & Et & 4-Me & $3 c$ & 95 & 96 \\
\hline 5 & 2-cyclohexenone & $\mathrm{CH}_{2} \mathrm{Ph}$ & $\mathrm{CH}_{2} \mathrm{Ph}$ & $\mathrm{H}$ & $3 a$ & 45 & $57(R)$ \\
\hline 6 & 2-cyclohexenone & $i$-Pr & $i-\operatorname{Pr}$ & $\mathrm{H}$ & 3a & 11 & $4(S)$ \\
\hline 7 & 2-cyclohexenone & \multicolumn{2}{|c|}{$-\mathrm{CH}_{2} \mathrm{CH}_{2} \mathrm{CH}_{2} \mathrm{CH}_{2} \mathrm{CH}_{2^{-}}$} & $\mathrm{H}$ & $3 a$ & 83 & $68(R)$ \\
\hline 8 & 2-cyclohexenone & \multicolumn{2}{|c|}{$-\mathrm{CH}_{2} \mathrm{CH}_{2} \mathrm{NCH}_{2} \mathrm{CH}_{2-}$} & $\mathrm{H}$ & 3a & 89 & $91(R)$ \\
\hline 9 & 2-cyclopentenone & Et & Et & $\mathrm{H}$ & $3 d$ & 95 & 75 \\
\hline 10 & 2-cyclopentenone & Et & Et & $3-\mathrm{Cl}$ & $3 \mathbf{e}$ & 98 & 81 \\
\hline 11 & 2-cyclopentenone & Et & Et & 3-MeO & $3 f$ & 97 & 95 \\
\hline 12 & 2-cycloheptenone & Et & Et & $\mathrm{H}$ & $3 g$ & 90 & 94 \\
\hline 13 & $(E)-\mathrm{C}_{5} \mathrm{H}_{11} \mathrm{CH}=\mathrm{CHCOCH}_{3}$ & Et & Et & $\mathrm{H}$ & $3 \mathbf{h}$ & 96 & 0 \\
\hline 14 & $(E)-\mathrm{C}_{5} \mathrm{H}_{11} \mathrm{CH}=\mathrm{CHCOCH}_{3}$ & $\mathrm{Me}$ & Et & $\mathrm{H}$ & $3 \mathbf{h}$ & 99 & $16(S)$ \\
\hline 15 & $(E)-\mathrm{C}_{5} \mathrm{H}_{11} \mathrm{CH}=\mathrm{CHCOCH}_{3}$ & $\mathrm{Me}$ & $n-\operatorname{Pr}$ & $\mathrm{H}$ & $3 \mathbf{h}$ & 95 & $24(S)$ \\
\hline 16 & $(E)-\mathrm{C}_{5} \mathrm{H}_{11} \mathrm{CH}=\mathrm{CHCOCH}_{3}$ & $\mathrm{Me}$ & $\mathrm{CH}_{2} \mathrm{Ph}$ & $\mathrm{H}$ & $3 \mathbf{h}$ & 94 & $10(S)$ \\
\hline 17 & $(E)-\mathrm{C}_{5} \mathrm{H}_{11} \mathrm{CH}=\mathrm{CHCOCH}_{3}$ & $\mathrm{Me}$ & $\mathrm{Ph}$ & $\mathrm{H}$ & $3 \mathbf{h}$ & 28 & $29(R)$ \\
\hline 18 & $(E)-\mathrm{C}_{5} \mathrm{H}_{11} \mathrm{CH}=\mathrm{CHCOCH}_{3}$ & Me & $i-\operatorname{Pr}$ & $\mathrm{H}$ & $3 \mathbf{h}$ & 94 & $35(R)$ \\
\hline
\end{tabular}


[a] All reactions were carried out at $50{ }^{\circ} \mathrm{C}$ for $6 \mathrm{~h}$ in the presence of enone $(1 \mathrm{mmol})$, arylboronic acid $(1.5 \mathrm{mmol})$, $[\mathrm{Rh} \text { (coe)Cl }]_{2}(0.015 \mathrm{mmol}, 3 \mathrm{~mol} \%), 4$ (0.066 mmol) and $\mathrm{KOH}(1 \mathrm{mmol})$ in dioxane- $\mathrm{H}_{2} \mathrm{O}(6 / 1)$.

[b] Isolated yields based on enones.

[c] Enantiomer excess determined by a chiral stationary column. 
Table 2. Effects of catalysts and bases ${ }^{[\mathrm{a}]}$

\begin{tabular}{cllccc}
\hline entry & rhodium complex & base & ${ }^{\circ} \mathrm{C} / \mathrm{h}$ & yield/\% & \%ee \\
\hline 1 & $1 / 2[\mathrm{RhCl}(\mathrm{coe})]_{2} / 5 \mathbf{a}$ & none & $50 / 16$ & 0 & - \\
2 & $1 / 2[\mathrm{RhCl}(\mathrm{coe})]_{2} / 5 \mathbf{a}$ & $\mathrm{Et}_{3} \mathrm{~N}$ & $50 / 16$ & 46 & $97(R)$ \\
3 & $1 / 2[\mathrm{RhCl}(\mathrm{coe})]_{2} / 5 \mathbf{a}$ & $\mathrm{K}_{2} \mathrm{CO}_{3}$ & $50 / 16$ & 26 & $98(R)$ \\
4 & $1 / 2[\mathrm{RhCl}(\mathrm{coe})]_{2} / 5 \mathbf{a}$ & $\mathrm{KOH}$ & $50 / 16$ & 84 & $98(R)$ \\
5 & {$\left[\mathrm{Rh}(\mathrm{nbd})_{2}\right] \mathrm{BF}_{4} / 5 \mathbf{a}$} & $\mathrm{Et}_{3} \mathrm{~N}$ & $50 / 16$ & 94 & $99(R)$ \\
6 & {$\left[\mathrm{Rh}(\mathrm{nbd})_{2}\right] \mathrm{BF}_{4} / 5 \mathbf{a}$} & $\mathrm{Et}_{3} \mathrm{~N}$ & $25 / 0.5$ & 99 & $99.6(R)$ \\
7 & {$\left[\mathrm{Rh}(\mathrm{nbd})_{2}\right] \mathrm{BF}_{4} / 5 \mathbf{b}$} & $\mathrm{Et}_{3} \mathrm{~N}$ & $25 / 2$ & 62 & $83(R)$ \\
8 & {$\left[\mathrm{Rh}(\mathrm{nbd})_{2}\right] \mathrm{BF}_{4} / 5 \mathrm{c}$} & $\mathrm{Et}_{3} \mathrm{~N}$ & $25 / 2$ & trace & - \\
\hline
\end{tabular}

[a] All reactions were carried out in the presence of 2-cyclohexenone (1 mmol), phenylboronic acid (1.5 mmol), rhodium(I) catalyst (3 mol\%), ligand (5, 3.3 mol\%), and base (if used, $1 \mathrm{mmol}$ ) in dioxane- $\mathrm{H}_{2} \mathrm{O}(6 / 1)$. 
Table 3. 1,4-Addition of arylboronic acid to $\alpha, \beta$-unsaturated carbonyl compounds catalyzed by a Rh(+)-5a complex ${ }^{[\mathrm{a}]}$

\begin{tabular}{|c|c|c|c|c|c|c|}
\hline entry & carbonyl compound & $\operatorname{ArB}(\mathrm{OH})_{2}, \mathrm{X}=$ & ${ }^{\circ} \mathrm{C} / \mathrm{h}$ & product No & yield/\% ${ }^{[\mathrm{b}]}$ & $\%$ eе ${ }^{[c]}$ \\
\hline 1 & 2-cyclohexenone & $\mathrm{H}$ & $25 / 0.5$ & 3a & 99 & $99.6(R)$ \\
\hline 2 & 2-cyclohexenone & 3-MeO & $25 / 2$ & $3 \mathbf{b}$ & 90 & $99.5(R)$ \\
\hline 3 & 2-cyclohexenone & $4-\mathrm{MeO}$ & $25 / 2$ & $3 \mathbf{i}$ & 99 & 99.8 \\
\hline 4 & 2-cyclohexenone & $3-\mathrm{Cl}$ & $25 / 2$ & $3 \mathbf{j}$ & 86 & 99.8 \\
\hline 5 & 2-cyclopentenone & $3-\mathrm{Cl}$ & $25 / 2$ & $3 \mathbf{e}$ & 99 & 96 \\
\hline 6 & 2-cyclopentenone & $4-\mathrm{MeO}$ & $25 / 2$ & $3 \mathbf{k}$ & 99 & 96 \\
\hline 7 & 2-cycloheptenone & $\mathrm{H}$ & $25 / 2$ & $3 g$ & 90 & 98 \\
\hline 8 & $(E)-\mathrm{C}_{5} \mathrm{H}_{11} \mathrm{CH}=\mathrm{CHCOCH}_{3}$ & $\mathrm{H}$ & $25 / 2$ & $3 \mathbf{h}$ & 87 & 74 \\
\hline 9 & $(E)-\mathrm{C}_{5} \mathrm{H}_{11} \mathrm{CH}=\mathrm{CHCOCH}_{3}$ & $\mathrm{H}$ & $5 / 48$ & $3 \mathbf{h}$ & 42 & 84 \\
\hline 10 & $(E)-\mathrm{C}_{5} \mathrm{H}_{11} \mathrm{CH}=\mathrm{CHCOCH}_{3}$ & 3-MeO & $25 / 2$ & $3 \mathbf{l}$ & 98 & 80 \\
\hline 11 & $(E)-\mathrm{C}_{5} \mathrm{H}_{11} \mathrm{CH}=\mathrm{CHCOCH}_{3}$ & 3-MeO & $5 / 48$ & $3 \mathbf{l}$ & 65 & 83 \\
\hline 12 & $(E)-\mathrm{C}_{5} \mathrm{H}_{11} \mathrm{CH}=\mathrm{CHCOCH}_{3}$ & $3-\mathrm{F}$ & $25 / 2$ & $3 \mathrm{~m}$ & 97 & 81 \\
\hline 13 & $(E)-\mathrm{C}_{5} \mathrm{H}_{11} \mathrm{CH}=\mathrm{CHCOPh}$ & 3-MeO & $25 / 2$ & $3 \mathbf{n}$ & 91 & 85 \\
\hline 14 & $(E)-\left(\mathrm{CH}_{3}\right)_{2} \mathrm{CHCH}=\mathrm{CHCOCH}_{3}$ & $\mathrm{H}$ & $25 / 6$ & 30 & 80 & $92(R)$ \\
\hline 15 & $(E)-\left(\mathrm{CH}_{3}\right)_{2} \mathrm{CHCH}=\mathrm{CHCOCH}_{3}$ & 3-MeO & $25 / 16$ & $3 \mathbf{p}$ & 78 & 94 \\
\hline 16 & $(E)-\left(\mathrm{CH}_{3}\right)_{2} \mathrm{CHCH}=\mathrm{CHCOCH}_{3}$ & $3-\mathrm{F}$ & $25 / 16$ & $3 \mathbf{q}$ & 71 & 90 \\
\hline 17 & $(E)-\left(\mathrm{CH}_{3}\right)_{2} \mathrm{CHCH}=\mathrm{CHCOC}_{6} \mathrm{H}_{11}$ & 3-MeO & $25 / 2$ & $3 \mathbf{r}$ & 62 & 81 \\
\hline 18 & $(E)-\left(\mathrm{CH}_{3}\right)_{2} \mathrm{CHCH}=\mathrm{CHCOPh}$ & 3-MeO & $25 / 6$ & $3 s$ & 98 & 85 \\
\hline 19 & $(E)-\mathrm{PhCH}=\mathrm{CHCOCH}_{3}$ & 3-MeO & $25 / 2$ & $3 t$ & 99 & 78 \\
\hline
\end{tabular}


[a] All reactions were carried out in the presence of enone $(1 \mathrm{mmol})$, arylboronic acid (1.5 mmol), $\left[\mathrm{Rh}(\mathrm{nbd})_{2}\right] \mathrm{BF}_{4}(0.03$ mmol, $3 \mathrm{~mol} \%)$, 5a (0.033 mmol) and $\mathrm{Et}_{3} \mathrm{~N}(1 \mathrm{mmol})$ in dioxane $(2.6 \mathrm{ml})$ and $\mathrm{H}_{2} \mathrm{O}(0.43 \mathrm{ml})$.

[b] Isolated yields based on enones.

[c] Enantiomer excess determined by a chiral stationary column. 


\section{Figure Captions Page}

Scheme 1. Asymmetric 1,4-addition of arylboronic acids to enones catalyzed by $\mathrm{Rh}(\mathrm{I})$-phosphoramidite complexes

Scheme 2. Phosphoramidites based on Linked-BINOL

Scheme 3. Asymmetric 1,4-addition to unsaturated esters

Figure 1. Amounts of catalyst loading and reaction rates

Table 1. 1,4-Addition of arylboronic acid to $\alpha, \beta$-unsaturated carbonyl compounds catalyzed by Rh(I)-4 complexes ${ }^{[\mathrm{a}]}$

Table 2. Effects of catalysts and bases ${ }^{[a]}$

Table 3. 1,4-Addition of arylboronic acid to $\alpha, \beta$-unsaturated carbonyl compounds catalyzed by a $\mathrm{Rh}(+)-5 a$ complex ${ }^{[\mathrm{a}]}$ 


\section{Table of Contents}

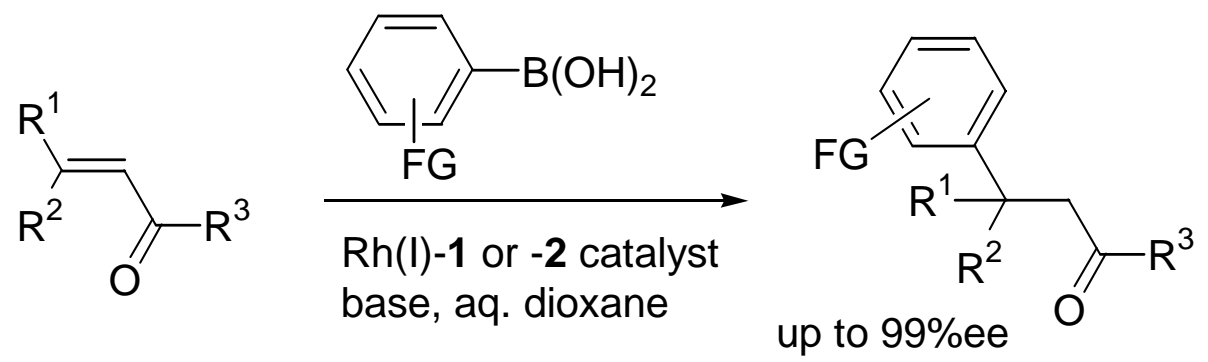<smiles>[R]N([R])p1oc2ccc3ccccc3c2c2c(ccc3ccccc32)o1</smiles>

1<smiles>[R]N([R])p1oc2ccc3ccccc3c2c2c(o1)c(COc1cc3ccccc3c3op(N([R])[R])oc4ccc5ccccc5c4c13)cc1ccccc12</smiles>

2: $\mathrm{R}=\mathrm{Me}, \mathrm{Et}, i-\mathrm{Pr}$

Chiral bidentate phosphoramidites (2) were newly synthesized for the rhodium(I)-catalyzed asymmetric 1,4 -addition of arylboronic acids to $\alpha, \beta$-unsaturated carbonyl compounds. The complex between $\left[\mathrm{Rh}(\mathrm{nbd})_{2}\right] \mathrm{BF}_{4}$ and $2(\mathrm{R}=\mathrm{Me})$ completed the addition to cyclic enones within $2 \mathrm{~h}$ at room temperature in the presence of $\mathrm{Et}_{3} \mathrm{~N}$ with $96-99.8 \%$ ee. The catalyst resulted in 66-94\%ee for acyclic enones. 